\title{
Dental Practitioner's Perception of the Compliance of Pediatric Patients to Orofacial Myotherapy Treatment Protocols: A Mixed Methods Study
}

\author{
Nikhita S Gune ${ }^{1}$, Amar N Katre ${ }^{2}$
}

\begin{abstract}
Introduction: The success of orofacial myotherapy in children is dependent on patient compliance to treatment protocols recommended by practitioners, such as reporting for regular follow-ups to the clinic, wear of appliances, and practice of orofacial myotherapy exercises at home. Due to the availability of limited literature on the same, this mixed methods study focused on studying the perception of Indian dental practitioners toward pediatric patient compliance to orofacial myotherapy treatment protocols.

Materials and methods: A self-administered, digital questionnaire was emailed to members of the Foundation of Orofacial Myotherapy. Participants were asked to rate the pediatric patient compliance in their practice on a 5-point Likert scale ranging from Very Compliant to Not Compliant at all. Parameters assessed included patient attendance for clinical follow-ups, regular appliance wear, and the daily practice of exercises at home. Qualitative data were collected based on the participant's answers to open-ended questions on compliance barriers faced by them in practice. Results: $39.5 \%$ of practitioners rated their patients to be compliant toward clinical follow-ups, while $27.9 \%$ of practitioners reported patients to be compliant to home schedule. Barriers to patient compliance reported by practitioners were lack of motivation of parent and child, absence of parental supervision, hectic patient schedule, peer pressure, and difficulty in performing exercises and wearing appliances.

Conclusion: Patient compliance to orofacial myotherapy treatment protocols remains a problem area for dental practitioners. There is a need to conduct future studies to explore behavioral interventions which can help practitioners overcome the barriers to patient compliance reported in this study.

Clinical significance: The results of this study can help practitioners who are already practicing orofacial myotherapy or plan to do so in future, to identify problem areas in their own practice, in the context of pediatric patient compliance toward orofacial myotherapy treatment protocols and the potential measures that can be implemented by them to overcome the same.

Keywords: Mixed methods study, Orofacial myotherapy, Patient compliance.

International Journal of Clinical Pediatric Dentistry (2021): 10.5005/jp-journals-10005-1909
\end{abstract}

\section{INTRODUCTION}

Orofacial myofunctional therapy (OMT) is an emerging branch of science in the fields of pediatric dentistry and medicine. It involves exercising the craniofacial muscles to improve proprioception, tone, and mobility to treat disorders of the stomatognathic system, such as mouth-breathing patterns, tongue-thrusting habits, improper joint and muscle patterns during speech, chewing and swallowing, and assist in the correction of parafunctional oral habits like thumb-sucking and bruxism in children. ${ }^{1}$ Orofacial myofunctional therapy exercises in such cases can help in establishing normal breathing and swallowing function and in improving the posture and movements of the tongue along with assisting in the proper development of the face and jaws. They can also contribute to improvement in body and head posture, gait, and prevention of certain respiratory ailments and general health conditions. ${ }^{2}$

Orofacial myofunctional therapy exercises are often used in combination with prefabricated or custom-made removable functional appliances in the treatment of orofacial myofunctional disorders. ${ }^{3}$ The success of such a protocol becomes heavily dependent on patient compliance toward regular clinical followups, daily wear of removable appliances at home, and performance of the exercises at home. Establishing compliance in pediatric patients is difficult as the dental practitioner must engage in effective communication with both parent and child. ${ }^{4}$ Pediatric patient non-compliance could prove to be the biggest drawback

\begin{abstract}
1Department of Pediatric Dentistry, NH SRCC Children's Hospital, Mumbai, Maharashtra, India

${ }^{2}$ Department of Pediatric and Preventive Dentistry, YMT Dental College and Hospital, Kharghar, Maharashtra, India

Corresponding Author: Nikhita S Gune, Department of Pediatric Dentistry, NH SRCC Children's Hospital, Mumbai, Maharashtra, India, Phone: +91 9833259339, e-mail: drnikhitagune@gmail.com

How to cite this article: Gune NS, Katre AN. Dental Practitioner's Perception of the Compliance of Pediatric Patients to Orofacial Myotherapy Treatment Protocols: A Mixed Methods Study. Int J Clin Pediatr Dent 2021;14(2):222-228.

Source of support: Nil

Conflict of interest: None
\end{abstract}

of this treatment protocol as it can lead to increased treatment duration or even abandonment and failure of the treatment.

A recent study estimated the patient compliance toward wearing prefabricated functional appliances to be as low as $30-31 \% .^{5}$ There is limited evidence available on pediatric patient compliance toward the performance of OMT exercises and wearing of prefabricated or custom-made removable functional appliances.

This study aimed to assess the dental practitioner's perception toward pediatric patient compliance to orofacial myotherapy treatment protocols in clinical practice and to explore practitioner 
reported barriers to compliance and measures taken to improve the same.

The objectives of this study were:

- To quantitatively assess the dental practitioner's perception of pediatric patient compliance toward regular clinical follow-up appointments and compare the same across years of OMT practice, number of ongoing cases, and type of practice.

- To quantitatively assess the dental practitioner's perception of pediatric patient compliance toward home schedule under the following parameters:

- Performing OMT exercises at home.

- Wearing prefabricated or custom-made functional appliances part-time during the day.

- Wearing prefabricated or custom-made functional appliances part-time during sleep hours

- To compare the same, across years of OMT practice, no of on-going cases and type of practice.

- To quantitatively and qualitatively analyze practitioner-reported factors for pediatric patient non-compliance toward follow-ups and home schedule and measures taken by them to improve the same.

\section{Materials and Methods}

This study was conducted in February 2020 and has been reported in accordance with the Good Reporting of A Mixed Methods Study (GRAMMS) checklist. All 102 life members of the Foundation of Orofacial Myotherapy (a charitable organization based in India) were invited to participate in the study. All the life members were registered dental practitioners in India and were dedicated to the learning and practice of this science. After taking their informed digital consent, a self-administered digital questionnaire was emailed to all the participants. The questionnaire included the demographic details of the participants including gender, age, years of OMT practice, number of ongoing/treated cases of OMT, and the type of practice such as specialty (i.e., pediatric dentistry or orthodontic practice)/consultant/general/institutional. The participants were also questioned about the frequency of clinical follow-ups conducted by them for their OMT patients and whether their practice had pre-decided days dedicated to these follow-ups.

The participant's perception toward patient compliance in their practice toward regular clinical follow-ups and a home schedule consisting of the performance of OMT exercises at home and regular wear of prefabricated/custom-made functional appliances part-time and during sleep hours was measured on a 5-point Likert scale. The Likert scale used was as follows: (1) Very compliant, (2) Compliant, (3) Neutral, (4) Not compliant, (5) Not compliant at all.

The barriers for patient non-compliance were measured quantitatively by asking participants to select the primary barrier (self-motivation/parental supervision/doctor-patient communication) they believed was the reason for non-compliance by patients and qualitatively by open-ended questions asking participants to enlist reasons reported by their patients for their non-compliance and measures taken by them to improve the same.

\section{Statistical Analysis}

All findings were recorded, and the data were captured into a Microsoft Office Excel (version 2013) spreadsheet. The master chart was checked for errors and discrepancies. Data analysis was done using windows based MedCalc Statistical Software version 13.3.1 (MedCalc Software by, Ostend, Belgium; http://www.medcalc.org; 2014). Descriptive statistics were used to detail the demographic characteristics of the participants such as age, gender, years of OMT practice, and number of ongoing/treated OMT cases.

Proportions were employed to measure patient compliance on the 5-point Likert scale to regular clinical follow-ups, home schedule inclusive of the performance of OMT exercises at home, regular wear of trainer appliances at home part-time and during sleep hours. Proportions were also used to measure the frequency of follow-ups and whether practitioners had pre-dedicated days for follow-up.

Practitioner's perception of pediatric patient compliance on the 5-point Likert scale toward regular clinical follow-ups and the home schedule was compared with respect to years of practice, number of ongoing/treated cases, and type of practice using the Chi-squared test.

Proportions were used to measure the practitioner's perception of the primary barrier for patient non-compliance. Qualitative data exploring practitioner reported reasons for patient non-compliance and measures taken to improve the same was transcribed, read, and re-read by the research team. Transcripts were subjected to thematic content analysis using coding. Original codes were assigned to mark themes identified in the transcripts that were relevant to the area of inquiry. Related codes were then grouped as major themes emerged from the data.

The qualitative and quantitative data were analyzed separately and in conjunction with each other to understand the practitionerreported compliance barriers specifically affecting either patient attendance to clinical follow-ups, the home schedule or both.

\section{Results}

\section{Demographic Distribution}

Table 1 provides an overview of the study population and outlines the distribution of participants w.r.t age, gender, years of OMT practice, type of practice and number of ongoing/treated OMT cases, frequency of clinical follow-ups conducted by them, and whether they had pre-decided days for OMT patients in their practice.

Out of the 102 participants included in the survey, 43 participants completed the survey. The response rate was $42.15 \%$.

$39.5 \%$ of the practitioners conducted clinical follow-ups once a month, while 18.6 and $30.2 \%$ conducted these follow-ups once a week and once in 2 weeks, respectively. $11.60 \%$ of participants conducted clinical follow-ups once in 3 months. $51.2 \%$ of practitioners had pre-decided days in their practice dedicated to conducting OMT patient follow-ups (Table 1).

\section{Practitioner's Perception of Patient Compliance to Regular Clinical Follow-ups}

$39.5 \%$ of practitioners rated that their patients were compliant to regular follow-ups, while $32.6 \%$ rated patient compliance to be neutral. $25.6 \%$ of practitioners rated that their patients were not compliant and $2.3 \%$ rated them to be not compliant at all. None of the practitioners rated patients to be very compliant to followups (Table 2).

\section{Practitioner's Perception of Patient Compliance to Home Schedule}

$41.9 \%$ of participants rated their patient compliance to home schedule to be neutral. $27.9 \%$ of practitioners rated that patients 
were compliant and not compliant equally to the home schedule. $2.3 \%$ of practitioners rated that their patients were not compliant at all to the home schedule (Table 2).

$44 \%$ of the practitioners reported that their patients perform OMT exercises at home 1-2 days a week, while $34.14 \%$ reported them to be performing the exercises 3-4 times a week. Only $9.75 \%$ reported them to be performing exercises every day, while $12.19 \%$ reported them to be not performing the exercises at home at all (Table 3).

$51.21 \%$ of practitioners reported that patients wore recommended appliances 3-4 times a week at night, while $29.2 \%$ reported them to be wearing it every night. $15 \%$ of the practitioners reported patients to be wearing the appliance 1-2 nights a week and $4.87 \%$ of practitioners reported them to be not wearing it at all during the night (Table 3 ).

Table 1: Demographic distribution of participants

\begin{tabular}{llcc}
\hline & & Frequency & Percentage \\
\hline Gender & Male & 14 & 32.55 \\
Age & Female & 29 & 67.44 \\
& 25-30 years & 21 & 48.8 \\
& 31-40 years & 15 & 34.9 \\
& 41-50 years & 5 & 11.6 \\
& >50 years & 2 & 4.7 \\
Years of OMT practice & Less than a year & 17 & 39.5 \\
& 1-2 years & 16 & 37.2 \\
Type of practice & 3-4 years & 6 & 14 \\
& >5 years & 4 & 9.3 \\
& Specialty & 22 & 53.5 \\
& Consultant & 9 & 20.9 \\
Number of ongoing/ & General & 5 & 11.6 \\
treated OMT cases & Institutional & 7 & 14 \\
& 11-20 cases & 29 & 67.4 \\
& 21-30 cases & 8 & 18.6 \\
& 31-50 cases & 1 & 4.7 \\
Frequency of clinical & Onc cases & 3 & 2.3 \\
follow-ups & Once a week & 8 & 7 \\
& Once in 2 weeks & 13 & 30.6 \\
& Once in 3 months & 17 & 39.5 \\
& & & 11.60 \\
\hline
\end{tabular}

$41.6 \%$ of practitioners reported patients to wearing the appliance part time during the day for 3-4 days a week, while $37 \%$ of practitioners reported that they wore it 1-2 days a week. $19.51 \%$ of practitioners reported patients wore it every day, while $2.43 \%$ reported they did not wear it all (Table 3).

Practitioner's perception of pediatric patient compliance on the 5-point Likert scale toward regular clinical follow-ups and the home schedule was compared with respect to years of practice, number of ongoing/treated cases, and type of practice using the Chi-squared test. However, there was no significant difference between the perception of the practitioners w.r.t. their years of OMT practice, number of ongoing/treated cases and type of practice (Table 4).

\section{Quantitative and Qualitative Analysis of Barriers Affecting Pediatric Patient Compliance and Measures Taken to Improve it}

$51 \%$ of the practitioners felt that absence of parental supervision was the primary barrier for pediatric patient non-compliance, while $39.5 \%$ of practitioners felt it was the absence of self-motivation and only $9.3 \%$ ascribed it to poor doctor-patient communication.

Qualitative analysis revealed that five major factors were acting as a barrier toward facilitating compliance among children. These included hectic school schedules, lack of parent and child motivation, absence of parental supervision, discomfort while wearing the appliance and performing exercises and peer pressure (Table 5).

Qualitative analysis of the measures used by practitioners to improve patient compliance revealed that the majority of the practitioners conducted regular follow-ups and provided reminder charts to children. Most of them conducted counseling sessions for both patients and parents. Some of them also conducted group therapy sessions at their clinic while a few used the behavior management technique "Modeling" to motivate their patients (Table 5).

\section{Discussion}

A patient's failure to follow the recommendations of his or her healthcare provider is a significant barrier that affects the successful treatment outcomes of many healthcare interventions. ${ }^{6}$ The same is true for orofacial myotherapy treatment protocols whose success is heavily dependent on patient compliance. With pediatric patients, the issue of compliance becomes two-fold as effective communication needs to be established with both parent and child, and both need to be motivated to ensure the child follows the exercise and appliance regime at home and reports to the clinic for

Table 2: Practitioner's perception of patient compliance to regular clinical follow-ups and home schedule

\begin{tabular}{llllll}
\hline & Very compliant & Compliant (\%) & Neutral (\%) & Not compliant (\%) & Not compliant at all (\%) \\
\hline Patient compliance to regular clinical follow-ups & 0 & 39.5 & 32.60 & 25.60 & 2.30 \\
Patient compliance to home schedule & 0 & 27.90 & 41.90 & 27.90 & 2.30 \\
\hline
\end{tabular}

Table 3: Practitioner's perception of patient compliance to performing OMT exercises at home, wearing appliances during sleep, and wearing appliances part-time during the day

\begin{tabular}{lllll}
\hline & $\begin{array}{l}\text { Every day } \\
(\%)\end{array}$ & $\begin{array}{l}3-4 \text { times a } \\
\text { week (\%) }\end{array}$ & $\begin{array}{l}\text { 1-2 days a } \\
\text { week (\%) }\end{array}$ & $\begin{array}{l}\text { Not wearing it } \\
\text { all (\%) }\end{array}$ \\
\hline Patient compliance to appliance wear at night & 29.20 & 51.21 & 15 & 4.87 \\
Patient compliance to appliance wear part-time during the day & 19.51 & 41.46 & 37 & 2.43 \\
Patient compliance to performing OMT exercises at home & 9.75 & 3.41 & 44 & 12.91 \\
\hline
\end{tabular}


Table 4: Assessment of practitioner's perception of pediatric patient compliance with respect to years of practice, number of ongoing/treated cases, and type of practice

\begin{tabular}{|c|c|c|c|c|c|c|}
\hline \multirow{2}{*}{$\begin{array}{l}\text { Patient compliance to } \\
\text { follow-up }\end{array}$} & \multicolumn{5}{|c|}{ Years of practice } & \multirow[b]{2}{*}{$p$ value } \\
\hline & $<1$ year & $1-2$ years & $3-5$ years & $>5$ years & & \\
\hline Very compliant (\%) & 0 & 0 & 0 & 0 & & 0.95 \\
\hline Compliant (\%) & 27.8 & 46.7 & 50.0 & 50.0 & & \\
\hline Neutral (\%) & 38.9 & 26.7 & 33.3 & 25.0 & & \\
\hline Not compliant (\%) & 27.8 & 26.7 & 16.7 & 25.0 & & \\
\hline \multirow[t]{3}{*}{ Not compliant at all (\%) } & 5.6 & 0 & 0 & 0 & & \\
\hline & \multicolumn{5}{|c|}{ No of ongoing/treated cases } & \\
\hline & $1-10$ & $11-20$ & $21-30$ & $31-50$ & $>50$ cases & \\
\hline Very compliant (\%) & 0 & 0 & 0 & 0 & 0 & 0.65 \\
\hline Compliant (\%) & 27.6 & 75.0 & 50.0 & 100.0 & 33.3 & \\
\hline Neutral (\%) & 34.5 & 25.0 & 50.0 & 0 & 33.3 & \\
\hline Not compliant (\%) & 34.5 & 0 & 0 & 0 & 33.3 & \\
\hline \multirow[t]{3}{*}{ Not compliant at all (\%) } & 3.4 & 0 & 0 & 0 & 0 & \\
\hline & \multicolumn{5}{|c|}{ Type of practice } & \\
\hline & Specialty & Consultant & General & Institutional & & \\
\hline Very compliant (\%) & 0 & 0 & 0 & 0 & & 0.18 \\
\hline Compliant (\%) & 59.1 & 33.3 & 0.0 & 16.7 & & \\
\hline Neutral (\%) & 13.6 & 33.3 & 60.0 & 66.7 & & \\
\hline Not compliant (\%) & 22.7 & 33.3 & 40.0 & 16.7 & & \\
\hline Not compliant at all (\%) & 4.5 & 0 & 0 & 0 & & \\
\hline \multirow{2}{*}{$\begin{array}{l}\text { Patient compliance to the } \\
\text { home schedule }\end{array}$} & \multicolumn{5}{|c|}{ Years of practice } & \\
\hline & $<1$ year & $1-2$ years & $3-5$ years & $>5$ years & & $p$ value \\
\hline Very compliant (\%) & 0 & 0 & 0 & 0 & & 0.81 \\
\hline Compliant (\%) & 22.2 & 40.0 & 33.3 & 0.0 & & \\
\hline Neutral (\%) & 38.9 & 40.0 & 50.0 & 50.0 & & \\
\hline Not compliant (\%) & 33.3 & 20.0 & 16.7 & 50.0 & & \\
\hline \multirow[t]{3}{*}{ Not compliant at all (\%) } & 5.6 & 0 & 0 & 0 & & \\
\hline & \multicolumn{5}{|c|}{ No of ongoing/treated cases } & \\
\hline & $1-10$ & $11-20$ & $21-30$ & $31-50$ & $>50$ cases & \\
\hline Very compliant (\%) & 0 & 0 & 0 & 0 & 0 & 0.91 \\
\hline Compliant (\%) & 20.7 & 50.0 & 50.0 & 0 & 33.3 & \\
\hline Neutral (\%) & 41.4 & 37.5 & 50.0 & 100.0 & 33.3 & \\
\hline Not compliant (\%) & 34.5 & 12.5 & 0.0 & 0 & 33.3 & \\
\hline \multirow[t]{3}{*}{ Not compliant at all (\%) } & 3.4 & 0 & 0 & 0 & 0 & \\
\hline & \multicolumn{5}{|c|}{ Type of practice } & \\
\hline & Specialty & Consultant & General & Institutional & & \\
\hline Very compliant (\%) & 0 & 0 & 0 & 0 & & 0.22 \\
\hline Compliant (\%) & 45.5 & 0 & 20.0 & 0 & & \\
\hline Neutral (\%) & 27.3 & 66.7 & 40.0 & 66.7 & & \\
\hline Not compliant (\%) & 22.7 & 33.3 & 40.0 & 33.3 & & \\
\hline Not compliant at all (\%) & 4.5 & 0 & 0 & 0 & & \\
\hline
\end{tabular}

regular follow-ups. ${ }^{4}$ There is limited published literature at present studying this aspect of orofacial myotherapy.

The response rate of this study was $42.5 \%$, as many practitioners were yet to adopt OMT protocols into their practice. For similar reasons, the demographic distribution of the participants revealed that a majority of them had been practicing OMT for less than a year or for 2 years and a majority of them had around 1-10 ongoing/ treated cases. Orofacial myotherapy is yet an emerging branch of science in India.

$39.5 \%$ of the participants kept clinical follow-ups at monthly intervals as this schedule would have been easier for their patients to incorporate in their routine compared with more rigorous 
Table 5: Analytical categories and keywords extracted from practitioner's response regarding barriers for pediatric patient non-compliance and measures to improve compliance

\begin{tabular}{|c|c|c|}
\hline Analytical categories & Themes & Keywords/quotes which were coded \\
\hline \multirow[t]{5}{*}{$\begin{array}{l}\text { Barriers causing patient non- } \\
\text { compliance }\end{array}$} & Hectic schedule of child & $\begin{array}{l}\text { School/tuition schedule, long hours of extracurricular activities, exams, } \\
\text { vacations }\end{array}$ \\
\hline & $\begin{array}{l}\text { Lack of motivation of par- } \\
\text { ent and child }\end{array}$ & $\begin{array}{l}\text { Child is not motivated, parents feel the treatment is unnecessary for child, } \\
\text { child cannot prioritize, child does not show interest, child cannot cope, child } \\
\text { forgets, lack of parental participation, child is lazy or bored }\end{array}$ \\
\hline & $\begin{array}{l}\text { Absence of parental } \\
\text { supervision }\end{array}$ & $\begin{array}{l}\text { Parents not at home, have long work hours, parents feel supervising children } \\
\text { is a waste of time, parents do not have time to supervise }\end{array}$ \\
\hline & Peer pressure & Patients feel ashamed as others will make fun of appliance, peer pressure \\
\hline & $\begin{array}{l}\text { Difficulty in doing exercises } \\
\text { or wearing the appliance }\end{array}$ & $\begin{array}{l}\text { Appliance hurts, difficult to perform exercises, cannot wear the appliance, } \\
\text { cannot understand exercise }\end{array}$ \\
\hline \multirow[t]{6}{*}{$\begin{array}{l}\text { Measures taken by practitioners } \\
\text { to improve patient compliance }\end{array}$} & $\begin{array}{l}\text { Patient schedule manage- } \\
\text { ment }\end{array}$ & $\begin{array}{l}\text { Fix a specific time for exercises, time management, specific day for follow- } \\
\text { ups, holiday for appointments }\end{array}$ \\
\hline & Motivational counseling & $\begin{array}{l}\text { Motivating patients, patient education, asking them to give equal impor- } \\
\text { tance to treatment, child counseling, tailored guidance }\end{array}$ \\
\hline & Modeling & $\begin{array}{l}\text { Show them bi-monthly changes, show them pre and post photos, showing } \\
\text { and interacting with other patients }\end{array}$ \\
\hline & Reminder therapy & $\begin{array}{l}\text { Diary and charts, reminder calls, reminder and activity charts, use of timers, } \\
\text { reminder messages, record-keeping related to appliance wear time }\end{array}$ \\
\hline & Regular follow-ups & See the kids every week, regular practice sessions \\
\hline & Group therapy & Group sessions \\
\hline
\end{tabular}

schedules of follow-ups once a week/once in 2 weeks. $51.2 \%$ of them dedicated specific days in their practice for OMT follow-ups to probably help establish a routine for themselves and their patients. $39.5 \%$ of practitioners rated patients to be compliant to clinical follow-ups compared with $27.9 \%$ of practitioners rating patient to be compliant to home schedule. This may be due to telephonic reminders sent by most practices to patients for follow-ups appointments as we noted in our qualitative analysis. In contrast, it may be difficult for practices to ensure patient adherence to home schedule. The percentage of practitioners rating patient's adherence to follow-ups and home schedule as non-compliant or non-compliant at all were of similar ranges. We included the neutral response in the Likert scale for participants who may be still at a learning curve in the field and may not yet have developed strong opinions on the same. In such a case, neutrality can help to avoid misleading responses.

Greater compliance was reported by practitioners to wearing prefabricated or custom-made functional appliances part-time during the day or during sleep hours compared with performing the exercises at home. This may be due to less physical effort required by patients to wear appliances in comparison to performing the exercises.

$51 \%$ of the practitioners felt that absence of parental supervision was one of the primary reasons for patient non-compliance. $40 \%$ felt absence of self-motivation was the primary barrier for patient non-compliance. Only $9 \%$ attributed non-compliance to difficulty in doctor-patient communication. Conducting a qualitative analysis revealed that themes other than those mentioned above could also be potential barriers for non-compliance. The barrier reported in the qualitative analysis were mainly directed toward patient compliance to the home schedule such as hectic schedule of the child, peer pressure and difficulty in performance of exercises and wearing the appliance, which is in accordance with our quantitative analysis, in which practitioners reported greater compliance to clinical follow-ups than the home schedule. Lack of motivation for child and parent and absence of parental supervision were common factors present in both qualitative and quantitative analysis. All of the barriers mentioned above are related to health behaviors and attitudes of pediatric patients and guardians.

The measures reported by practitioners for improving compliance in their practice were also primarily directed at improving patient compliance to the home schedule. Practitioners focused more on the use of reminder therapy and patient schedule management to achieve the same, rather than implementing behavioral intervention techniques. Few practitioners did attempt to bring about behavior changes by use of modeling and motivational counseling sessions, etc.

There is a necessity to examine behavioral interventions used in weight loss and tobacco cessation practices, which typically struggle with achieving patient compliance but have now achieved considerable success. ${ }^{7}$ The same could be adapted to OMT practices.

The limitations of this study include the use of a non-validated scale in the questionnaire as there are no previously published studies studying this aspect of orofacial myotherpy. Also, the use of a self-report scale could have led to biased responses. Observational studies directly measuring patient compliance could be conducted with the use of phone-based applications or reminder charts, wherein the patients themselves mark their attendance to OMT protocols. Long-term observational studies examining the effect of behavioral interventions on patient compliance to OMT protocols should be also conducted in the future.

The field of orofacial myotherapy has the potential to positively affect the quality of life of children suffering from myofunctional disorders; however, patient compliance needs to be ensured by practitioners for achieving successful outcomes. 


\section{Conclusion}

$39.5 \%$ of practitioners reported their patients to be compliant to regular clinical follow-ups compared with $27.9 \%$ of practitioners reporting patients to be compliant to home schedule. Greater compliance among patients was reported by practitioners toward wearing appliances compared with performing the OMT exercises at home. Barriers to patient compliance included lack of motivation of parent and child, absence of parental supervision, hectic patient schedule, peer pressure, and difficulty in performing exercises and wearing appliance. There is an urgent need to conduct further studies in this field, which can help practitioners develop standardized OMT protocols to ensure patient compliance.

\section{Clinical Significance}

The results of this study can help practitioners who are already practicing orofacial myotherapy or plan to do so in future, to identify problem areas in their own practice, in the context of pediatric patient compliance toward orofacial myotherapy treatment protocols and the potential measures that can be implemented by them to overcome the same.

\section{References}

1. Homem MA, Vieira-Andrade RG, Falci SG, et al. Effectiveness of orofacial myofunctional therapy in orthodontic patients: a systematic review. Dental Press J Orthod 2014;19(4):94-99. DOI: 10.1590/21769451.19.4.094-099.oar.

2. Acharya P, Jawdekar A, Orofacial Functional Myotherapy. IDRR. May, 2017. Available at: https://www.idrr.in/article/print/103. (Last accessed on 12, July, 2019).

3. Idris G, Hajeer MY, Al-Jundi A. Soft- and hard-tissue changes following treatment of class II division 1 malocclusion with activator versus trainer: a randomized controlled trial. Eur J Orthod 2019;41(1):21-28. DOI: 10.1093/ejo/cjy014.

4. Wright GZ, Kupietzky A, ed. Behavior management in dentistry for children. Ames: John Wiley \& Sons; 2014. p. 79.

5. Cirgic E, Kjellberg H, Petzold M, et al. A cost-minimization analysis of large overjet reduction with two removable functional appliances based on a randomized controlled trial. Eur J Orthod 2017;40(4):437443. DOI: 10.1093/ejo/cjx077.

6. Martin LR, Williams SL, Haskard KB, et al. The challenge of patient adherence. Ther Clin Risk Manag 2005;1(3):189-199.

7. Hooker S, Punjabi A, Justesen K, et al. Encouraging health behavior change: eight evidence-based strategies. Fam Pract Manag 2018;25(2):31-36. 


\section{Annexure 1: Questionnaire Demographic Details}

1. Age: $25-35 / 36-45 / 46-55 /$ more than 55 years

2. Gender: Male/Female

3. Number of years of OMT practice: Less than a year/1-2 years/3-4 years/more than 5 years

4. Type of orofacial myotherapy practice: Specialty/General/ Consultant/Institutional

5. Number of ongoing/treated OMT cases: 1-10 cases/11-20 cases/21-30/31-50/more than 50 cases

6. What is the frequency of follow-up appointments you recommend to patients undergoing orofacial myotherapy?

- Once a week

- Once in 2 weeks

- Once a month

- Once in 3 months

7. Are there specific pre-decided days and sessions in your practice to conduct follow-up sessions with your orofacial myotherapy patients?

- Yes

- No

\section{Questions}

1. How would you rate the patient compliance in your practice to the follow-up schedule recommended by you?

- Very compliant

- Compliant

- Neutral

- Not compliant

- Not compliant at all

2. How would you rate the patient compliance in your practice to the home schedule recommended by you?

- Very compliant

- Compliant
- Neutral

- Not compliant

- Not compliant at all

3. What is the wearing frequency of trainer appliances selfreported by your patients during sleep?

- Every night

- 3-4 nights a week

- 1-2 nights a week

- Never wears at night

4. What is the wearing frequency of trainer appliances selfreported by patients during the day?

- Every day

- 3-4 days a week

- 1-2 days a week

- Never wears during the day

5. In what frequency do patients self-report performing OMT exercises at home?

- Every day

- 3-4 days a week

- 1-2 days a week

- Never done at home

6. What is the primary barrier for non-compliance of patients to OMT protocols?

- Absence of parental supervision

- Absence of self-motivation

- Poor doctor-patient communication

7. What are the reasons reported to you by patients/parents for non-compliance to OMT protocols and state any measures you have adopted in your practice to improve patient compliance? 